\title{
COVID-19 Vaccination: An Overview and Education Tool for Nuclear Medicine Technologists
}

\author{
L. Harold Barnwell III, DNAP, CRNA, and Mark H. Crosthwaite, CNMT, FSNMMI-TS \\ Virginia Commonwealth University, Richmond, Virginia
} CE credit: For CE credit, you can access the test for this article, as well as additional JNMT CE tests, online at https://www.snmmilearningcenter.org.
Complete the test online no later than March 2024 . Your online test will be scored immediately. You may make 3 attempts to pass the test and must
answer $80 \%$ of the questions correctly to receive 1.0 CEH (Continuing Education Hour) credit. SNMMI members will have their CEH credit added to their
VOICE transcript automatically; nonmembers will be able to print out a CE certificate upon successfully completing the test. The online test is free to SNMMI
members; nonmembers must pay $\$ 15.00$ by credit card when logging onto the website to take the test.

Some nuclear medicine technologists may be called on to administer COVID-19 vaccines, but many may have never administered a vaccine. This article summarizes COVID-19 vaccination procedures and provides links to additional information, training, and self-assessment.

Key Words: CNMT; infectious disease; COVID-19; IM administration; intramuscular injection; standards of practice for NMT

J Nucl Med Technol 2021; 49:7-10

DOI: $10.2967 /$ jnmt.121.261921

$\mathbf{H}$ ealth-care systems nationwide are struggling with the logistical challenges of administering vaccinations against coronavirus disease 2019 (COVID-19) (1). On January 5, 2021 , less than $30 \%$ of the $15,418,500$ COVID-19 vaccine doses distributed to states had been administered, with only $4,563,260$ individuals receiving the first of 2 required vaccine injections (2). One barrier to vaccine administration has been the shortage of available health-care professionals trained to administer the vaccine (1). One solution to the problem proposes the use of health-care professionals other than nurses to provide injections. Several health systems have already implemented the Centers for Disease Control and Prevention (CDC) contingency strategy for mitigating staffing shortages by asking health-care providers to support patient care in areas outside their usual work setting (3).

The Society of Nuclear Medicine and Molecular Imaging Technologist Section COVID-19 Task Force recognizes that some nuclear medicine technologists (NMTs) may be called on to administer COVID-19 vaccines, as it is a skill

\footnotetext{
Received Jan. 11, 2021; revision accepted Jan. 13, 2021.

For correspondence and reprints contact: Mark $\mathrm{H}$. Crosthwaite, Virginia Commonwealth University, 701 W. Grace St., Suite 2100, P.O. Box 843057, Richmond, VA 23284.

E-mail: mhcrosthwait@vcu.edu

Published online Jan. 22, 2021.

COPYRIGHT (c) 2021 by the Society of Nuclear Medicine and Molecular Imaging.
}

incorporated within the NMT scope of practice (4). Some of these same technologists may be uncomfortable giving intramuscular injections, and many, if not all, NMTs may have never administered a vaccine. For this reason, the CDC has created an online COVID-19 vaccination training program and reference materials to assist inexperienced health-care professionals (5). This article summarizes COVID19 vaccination procedures and provides links to additional information, training, and self-assessment.

\section{TRAINING RESOURCES}

Health-care providers proactively preparing to administer COVID-19 vaccines will benefit from a refresher on vaccine administration and medical information about the COVID-19 vaccine. The CDC launched a series of web-based, self-paced electronic learning modules (6). Three modules with text and images (no audio or video) are currently available: "COVID-19 Vaccine Training: General Overview of Immunization Best Practices for Healthcare Providers" (7); "Moderna COVID-19 Vaccine: What Healthcare Professionals Need to Know" (8); and "Pfizer-BioNTech COVID-19 Vaccine: What Healthcare Professionals Need to Know" (9).

NMTs planning to administer COVID-19 vaccines are encouraged to complete these modules in their entirety. On completion of the modules, technologists can print a continuing education certificate worth $1.25 \mathrm{~h}$. The Accreditation Council for Pharmacy Education, one of the organizations offering continuing education, is recognized by the Nuclear Medicine Technology Certification Board (7-9).

\section{ABOUT COVID-19 VACCINES}

COVID-19 vaccination efforts began nationwide in December 2020 after the U.S. Food and Drug Administration authorized emergency use of the Pfizer-BioNTech and Moderna vaccines. Both vaccines are messenger RNA (mRNA) vaccines (7-9). Although clinical use of this type of vaccine is relatively new, it is a product of research and development spanning decades (10).

The 2 approved COVID-19 mRNA vaccines do not incorporate the live virus, severe acute respiratory syndrome 
TABLE 1

Intramuscular Injection Technique and Supplies in Adults at Least 19 Years Old (14)

\begin{tabular}{|c|c|}
\hline Step & Description \\
\hline 1 & Perform proper hand hygiene \\
\hline 2 & Use aseptic technique when handling, preparing, and administering vaccine \\
\hline 3 & Remove vaccine from storage unit \\
\hline 4 & Verify that correct vaccine has been selected \\
\hline 5 & Check expiration date (for Moderna vaccine, scan matrix barcode on vial (8)) \\
\hline 6 & $\begin{array}{l}\text { Gather supplies: syringe ( } 1 \text { or } 3 \mathrm{~mL}) \text {, needle }\left(16,25 \text {, or } 38 \mathrm{~mm}\left[5 / 8,1 \text {, or } 11 \frac{1}{2} \mathrm{in}\right]^{\star} ; 22-25 \mathrm{~g}\right) \text {, sterile alcohol wipes, adhesive } \\
\text { bandages, gauze, and medication label }\end{array}$ \\
\hline 7 & Identify administration site: deltoid muscle of upper arm (preferred) or vastus lateralis muscle of anterolateral thigh \\
\hline
\end{tabular}

*For deltoid administration, recommended gauge 22 to 25 ; for anyone $<130 \mathrm{lb}$ or $<59 \mathrm{~kg}, 16-25 \mathrm{~mm}(5 / 8$ to $1 \mathrm{in})$ needle, or $130-152 \mathrm{lb}$ or 59-69 kg, $25 \mathrm{~mm}$ (1 in) needle; for women 152-200 lb or 69-91 kg or for men 152-260 lb or 69-118 kg, 25-38mm (1 to 1.5 in) needle; for women $>200 \mathrm{lb}$ or $>91 \mathrm{~kg}$ or for men $>260 \mathrm{lb}$ or $>118 \mathrm{~kg}, 38 \mathrm{~mm}$ or (1.5 in) needle.

coronavirus 2 (SARS-CoV-2) (10). Therefore, one cannot contract COVID-19 from the vaccine $(8,9)$. Instead, the vaccine instructs the body's cells to create a single harmless protein unique to the virus. The protein mimics the spike-shaped surface protein of the SARS-CoV-2. The immune system detects the protein spike and recognizes that it does not belong there, triggering the immune system to create memory T-lymphocyte and B-lymphocyte antibodies that will recognize and fight SARS-CoV-2 and prevent future infection. The vaccinated material does not enter the cell nucleus to interact with genetic material. Once the cell produces the spike protein, the immune system destroys the injected vaccine's instructions to make the protein (10).

Because of severe health risks associated with COVID19 and because reinfection with COVID-19 is possible, the CDC recommends that individuals with prior COVID-19 should be offered the vaccination and encouraged to accept it (11). However, those with active COVID-19 should defer vaccination until after recovery $(8,9)$. Additional vaccination considerations include individuals with immunocompromising conditions, including HIV; those taking immunosuppressive medications; and those who are pregnant or lactating.

\section{PFIZER-BIONTECH VACCINE}

Each 2-mL multidose vial of the Pfizer-BioNTech vaccine contains 6 doses and requires dilution with preservative-free normal saline (9). The recommended dose is $0.3 \mathrm{~mL}$ administered intramuscularly on 2 separate occasions, $21 \mathrm{~d}$ apart. After both doses have been administered, the vaccine effectively prevents COVID-19 in $92 \%$ of recipients. Contraindications include a severe allergic reaction (e.g., anaphylaxis) to any vaccine component or to a previous dose of the PfizerBioNTech vaccine. The Pfizer emergency-use authorization lists the vaccine ingredients (11). Precautions are advised in patients who have had a severe allergic reaction to any other vaccine or injectable therapy (intramuscular, intravenous, or subcutaneous) or have moderate to severe acute illness (9).

\section{MODERNA VACCINE}

Each 5-mL multidose vial of the Moderna vaccine contains 10 doses in a preservative-free sterile liquid (8). No diluent is required. The recommended dose is $0.5 \mathrm{~mL}$ administered intramuscularly on 2 separate occasions, $28 \mathrm{~d}$ apart. After both doses have been administered, the vaccine effectively prevents COVID-19 in 94\% of recipients. Contraindications include a severe allergic reaction (e.g., anaphylaxis) to a previous dose or component of either mRNA COVID-19 vaccine. The Moderna emergency-use authorization lists the vaccine ingredients (12). Precautions are advised in patients who have a history of an immediate allergic reaction to any other vaccine or injectable therapy (intramuscular, intravenous, or subcutaneous vaccines or therapies not related to a component of the mRNA COVID-19 vaccines) or have moderate to severe acute illness (8).

\section{ANAPHYLAXIS}

Rare cases of anaphylaxis, an acute life-threatening allergic reaction, have been reported after COVID-19 vaccine

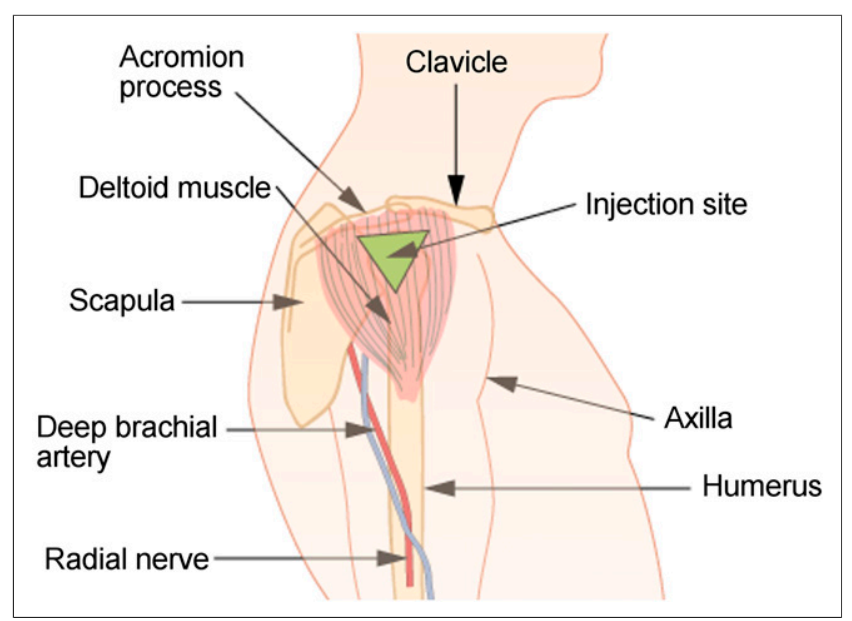

FIGURE 1. Intramuscular injection site: deltoid muscle and related anatomic landmarks (https://en.wikipedia.org/wiki/lntramuscular_ injection) (19). 
TABLE 2

Strategies to Reduce Anxiety and Pain Associated with Injections (18)

\begin{tabular}{ll}
\hline \multicolumn{1}{c}{ Strategy } & Description \\
\hline Positive attitude & Display a positive attitude through facial expressions, body language, and comments \\
Soft voice & Use a soft and calm tone of voice \\
Eye contact & Look the patient in the eye \\
Explanations & is a shield to protect your body against infection") \\
Bonesty & Be honest in explaining what to expect (e.g., do not say, "this injection won't hurt") \\
\hline
\end{tabular}

administration $(8,9)$. Per the CDC, appropriate medical treatment for severe allergic reactions must be immediately available in the event that an acute anaphylactic reaction occurs after administration of an mRNA COVID-19 vaccine (13). For this reason, the CDC recommends monitoring any individual with a history of anaphylaxis, regardless of the cause, for 30 min after COVID-19 vaccination. Everyone else should be monitored for $15 \mathrm{~min}$ after COVID-19 vaccination. A comprehensive discussion of the medical management of anaphylaxis is outside the scope of this article. The CDC recommends that all vaccination centers have a written protocol, equipment, and medications to manage medical emergencies after vaccination $(8)$.

\section{STORAGE AND HANDLING}

Both the Pfizer-BioNTech and the Moderna COVID-19 vaccines are shipped and stored at extremely low temperatures requiring temperature monitoring until administration $(8,9)$. The Pfizer-BioNTech vaccine arrives packaged in dry ice. The CDC provides a 1-page dry-ice safety document for health-care professionals. The Pfizer-BioNTech vaccine can be refrigerated at $2^{\circ} \mathrm{C}-8^{\circ} \mathrm{C}\left(36^{\circ} \mathrm{F}-46^{\circ} \mathrm{F}\right)$ for up to $5 \mathrm{~d}(120 \mathrm{~h})$ before administration (9). The Moderna vaccine can be refrigerated at $2^{\circ} \mathrm{C}-8^{\circ} \mathrm{C}\left(36^{\circ} \mathrm{F}-46^{\circ} \mathrm{F}\right)$ for up to $30 \mathrm{~d}$ before administration $(8)$.

\section{PREPARATION}

After hand hygiene has been applied, the expiration date of the vaccine (and of the diluents for the Pfizer-BioNTech vaccine) must be checked before preparation of the medication, which should be done in a clean area using strict aseptic technique $(8,9)$. Each vial of the Pfizer-BioNTech vaccine must be diluted with $1.8 \mathrm{~mL}$ of $0.9 \%$ sodium chloride (9). A module from the CDC (9) provides detailed instructions for mixing the vaccine and diluent. The Moderna vaccine does not require a diluent (8). Once prepared, both the PfizerBioNTech and the Moderna vaccines should be kept at room temperature (i.e., $2^{\circ} \mathrm{C}-25^{\circ} \mathrm{C}\left[35^{\circ} \mathrm{F}-77^{\circ} \mathrm{F}\right]$ ) and administered within $6 \mathrm{~h}$ of preparation (9). Any mixed, unused vaccine (Pfizer-BioNTech and Moderna) must be discarded after 6 h $(8,9)$.

\section{ADMINISTRATION}

Although intramuscular injections are within the NMT's scope of practice, they may not be part of a technologist's routine clinical practice. The CDC has produced a video (14) providing a refresher on administering intramuscular injections to adults (15). Table 1 summarizes the information presented in the video, and Figure 1 illustrates the deltoid muscle and the preferred intramuscular injection site.

The CDC recommends wearing personal protective equipment, including a face covering, when administering COVID-19 vaccines $(8,9)$. The vaccination process includes gathering the necessary supplies for the vaccination and then identifying and welcoming the patient and establishing a rapport (16). The patient should be provided with specific information about the vaccination process, which vaccine is to be administered, and the type of injection (e.g., deltoid intramuscular). Before administering the vaccine, it is important to review the patient's COVID-19 vaccine history, screen for contraindications and precautions, and give the patient a copy of the emergency-use authorization fact sheet for recipients and caregivers for the respective vaccine $(8,9,12,13)$.

TABLE 3

Rights of Medication Administration (17)

\begin{tabular}{ll}
\hline Right & \\
\hline 1 & Right patient \\
2 & Right vaccine and diluent (if diluent is applicable, such as for Pfizer-BioNTech vaccine) \\
3 & Right time (including correct age and interval, as well as use before product expiration time/date) \\
4 & Right dosage \\
5 & Right route (including correct needle gauge and length and correct technique) \\
7 & Right site \\
\hline
\end{tabular}


Administration of the dose requires applying hand hygiene and donning clean gloves before identifying the injection site $(8,9)$. The center of the deltoid muscle is the recommended injection site for COVID-19 vaccination in adults. This site, located approximately $5 \mathrm{~cm}$ (2 in) below the acromion process on the lateral side of the shoulder above the axillary fold (i.e., armpit), is the thickest part of the deltoid muscle (Fig. 1). The skin over the injection site is cleaned with a sterile alcohol wipe using a circular motion from the center to a circle $5-7.5 \mathrm{~cm}(2-3 \mathrm{in})$ in diameter, and the alcohol is allowed to dry (12). While the patient's arm is stabilized with the nondominant hand, the needle is quickly inserted into the injection site at a $90^{\circ}$ angle, and the entire dose is injected. The needle is withdrawn at the same $90^{\circ}$ angle as inserted $(8,9,12)$. The syringe should not be aspirated before or after the injection (16). After removal of the needle, gentle pressure is applied over the injection site with a clean gauze for several seconds, or an adhesive bandage is applied (12). Use of strategies to reduce the patient's anxiety and the pain associated with the injection is recommended (Table 2) (16). Finally, the vaccination must be documented, including the date, lot number, vaccine manufacturer, administration site, education materials provided to the patient, and provider's name or initials (12).

The CDC's best practices for vaccine administration include following the "Rights of Medication Administration" (Table 3) (17), providing staff training and education, and using a checklist to assess vaccine administration skills (18). A skills checklist for vaccine administration is available for health-care professionals to self-assess vaccine administration competency in 5 domains: patient education, medical protocols, vaccine preparation, vaccine administration, and documentation procedures (16).

\section{SUMMARY}

As health-care systems face the logistical challenges of COVID-19, the strategy of using all competent health-care providers to support patient care in areas outside their usual work setting seems likely. Specifically, to address the shortage of available health-care providers trained to administer vaccines, NMTs may be asked to administer COVID-19 vaccinations. Doing so may be the first clinical experience some NMTs have in administering a vaccine. This article offers NMTs a summary of the procedure and provides several resources to proactively prepare to administer COVID-19 vaccinations. NMTs who will be administering COVID-19 vaccines are encouraged to complete the recommended CDC modules in their entirety. The spectrum of responsibilities for a NMT varies widely across the United States. Information presented in this article provides a basis for establishing knowledge and understanding related to the intramuscular injection of COVID-19 vaccines that may be performed by a NMT. NMTs must comply with all federal, state, and institutional guidelines, including proper documentation of competency in the practice of this activity.

\section{DISCLOSURE}

No potential conflict of interest relevant to this article was reported.

\section{REFERENCES}

1. Robins R, Robles F, Arango T. Here's why distribution of the vaccine is taking longer than expected. New York Times website. https://www.nytimes.com/2020/ 12/31/health/vaccine-distribution-delays.html?auth=login-email\&login=email. Published December 31, 2020. Updated January 11, 2021. Accessed January 15, 2021.

2. CDC COVID data tracker. Centers for Disease Control and Prevention website. https://covid.cdc.gov/covid-data-tracker/\#vaccinations. Accessed January 15, 2021.

3. Strategies to mitigate healthcare personnel staffing shortages. Centers for Disease Control and Prevention website. https://www.cdc.gov/coronavirus/2019-ncov/ hcp/mitigating-staff-shortages.html. Updated December 14, 2020. Accessed January $15,2021$.

4. SNMMI-TS Scope of Practice Task Force. Nuclear medicine technologist scope of practice and performance standards. J Nucl Med Technol. 2017;45:325-336

5. COVID-19 vaccination training programs and reference materials for healthcare professionals. Centers for Disease Control and Prevention website. https://www. cdc.gov/vaccines/covid-19/downloads/COVID-19-Clinical-Training-and-Resourcesfor-HCPs.pdf. Published November 24, 2020. Accessed January 15, 2021.

6. COVID-19 vaccine training modules. Centers for Disease Control and Prevention website. https://www2.cdc.gov/vaccines/ed/covid19/. Updated December 10, 2020. Accessed January 15, 2021.

7. CE instructions for WB4460: COVID-19 vaccine training: general overview of immunization best practices for healthcare providers. Centers for Disease Control and Prevention website. https://www2.cdc.gov/vaccines/ed/covid19/SHVA/ index.asp. Published December 15, 2020. Accessed January 15, 2021

8. CE instructions for WB4464: Moderna COVID-19 vaccine: what healthcare professionals need to know. Centers for Disease Control and Prevention website. https://www2.cdc.gov/vaccines/ed/covid19/moderna/index.asp. Published December 28, 2020. Accessed January 15, 2021.

9. CE instructions for WB4461: Pfizer-BioNTech COVID-19 vaccine: what healthcare professionals need to know. Centers for Disease Control and Prevention website. https://www2.cdc.gov/vaccines/ed/covid19/pfizer/index.asp. Published December 16, 2020. Accessed January 15, 2021.

10. Understanding mRNA COVID-19 vaccines. Centers for Disease Control and Prevention website. https://www.cdc.gov/coronavirus/2019-ncov/vaccines/differentvaccines/mrna.html. Updated December 18, 2020. Accessed January 15, 2021.

11. Facts about COVID-19 vaccines. Centers for Disease Control and Prevention website. https://www.cdc.gov/coronavirus/2019-ncov/vaccines/facts.html. Updated January 4, 2021. Accessed January 15, 2021.

12. Fact sheet for recipients and caregivers: emergency use authorization (EUA) of the Pfizer-BioNTech COVID-19 vaccine to prevent coronavirus disease 2019 (COVID-19) in individuals 16 years of age and older. Food and Drug Administration website https://www.fda.gov/media/144414/download. Updated December 2020. Accessed January 15, 2021.

13. Fact sheet for recipients and caregivers: emergency use authorization (EUA) of the Moderna COVID-19 vaccine to prevent coronavirus disease 2019 (COVID-19) in individuals 18 years of age and older. Moderna website. https://www.modernatx.com/covid19vaccine-eua/eua-fact-sheet-recipients.pdf. Updated December 2020. Accessed January 15, 2021.

14. Intramuscular (IM) injection: supplies-adults 19 years and older [video]. Centers for Disease Control and Prevention website. https:/www2.cdc.gov/vaccines/ ed/vaxadmin/VA_Video/imsupplies/imsupplies.html. Accessed January 15, 2021.

15. Dry ice safety for healthcare professionals. Centers for Disease Control and Prevention website. https://www.cdc.gov/vaccines/covid-19/info-by-product/pfizer/downloads/dry-ice-safety-hcp.pdf. Published January 7, 2021. Accessed January 15, 2021.

16. Skills checklist for vaccine administration. Immunization Action Coalition website. https://www.immunize.org/catg.d/p7010.pdf. Updated August 2020. Accessed January 15, 2021.

17. Vaccine administration. Centers for Disease Control and Prevention website. https://www.cdc.gov/vaccines/pubs/pinkbook/downloads/vac-admin.pdf. Accessed January $15,2021$.

18. Vaccine administration [slide show]. Centers for Disease Control and Prevention website. https://www2.cdc.gov/vaccines/ed/vaxadmin/va/index.html. Accessed January 15, 2021.

19. Intramuscular injection. Wikipedia website. https://en.wikipedia.org/wiki/Intramuscular_injection. Updated January 7, 2021. Accessed January 27, 2021. 